\title{
WIF1 prevents Wnt5A mediated LIMK/CFL phosphorylation and adherens junction disruption in human vascular endothelial cells
}

\author{
Tom Skaria ${ }^{1}$, Esther Bachli ${ }^{2}$ and Gabriele Schoedon ${ }^{1 *}$
}

\begin{abstract}
Background: Wnt5A is released by activated macrophages and elevated levels have been detected in sepsis patients with severe systemic inflammation. However, the signalling and functional effects of Wnt5A in the vascular endothelial cells (VEC) remained unclear. Recently, we showed that Wnt5A affects barrier function in human VEC through Ryk interaction. Wnt5A/Ryk signalling activates LIMK to inactivate the actin depolymerisation factor CFL by phosphorylation, promotes actin polymerisation and disrupts endothelial adherens junctions.

Findings: Here, we investigate the antagonistic effect of the Ryk specific secreted Wnt antagonist Wnt inhibitory factor (WIF)-1 on Wnt5A-mediated activation/inactivation of LIMK/CFL, and adherens junction disruption in human VEC. In human coronary artery endothelial cells (HCAEC), treatment with Wnt5A enhanced the phosphorylation of LIMK and CFL that was significantly prevented by WIF1. The presence of WIF1 suppressed Wnt5A-mediated disruption of $\beta$-catenin and VE-cadherin adherens junctions in HCAEC, thereby preventing barrier dysfunction caused by Wnt5A.

Conclusion: We conclude that WIF1 or molecules with similar properties could be potent tools for the prevention of vascular leakage due to Wnt5A-mediated actin cytoskeleton remodeling in diseases associated with systemic inflammation.
\end{abstract}

Keywords: Inflammation, Endothelial permeability, Wnt5A, WIF1, Ryk, Cytoskeleton remodeling

\section{Background}

Vascular endothelial cells (VEC) in physiologic state play a crucial role in maintaining vascular homeostasis. They preserve blood fluidity $[1,2]$, control blood pressure, prevent the adherence of leukocytes [1] and platelets [2], and regulate vascular permeability [1]. In diseases associated with inflammatory states, vascular leakage is a major pathophysiologic event. In systemic inflammation and sepsis, as well as in autoimmune and allergic inflammation, activated blood components and inflammatory mediators such as cytokines act upon VEC and heavily alter their functions [2], conferring an inflamed phenotype.

\footnotetext{
*Correspondence: klinsog@usz.uzh.ch

${ }^{1}$ Inflammation Research Unit, Division of Internal Medicine, University

Hospital Zürich, Rämistrasse 100, CH-8091 Zürich, Switzerland

Full list of author information is available at the end of the article
}

Inflamed VEC are procoagulant and exhibit increased production of vasoactive substances, expression of cell adhesion molecules, synthesis of inflammatory mediators including chemoattractants, and barrier dysfunction causing leakage [1].

Wnt5A, a lipid modified signalling protein of the noncanonical Wnt family [3] is detected in high levels in sera and bone marrow of patients with severe systemic inflammation and septic shock $[4,5]$. Its expression has also been found elevated in macrophage-accumulated intima from human and murine atherosclerotic lesions [6]. Wnt5A is a chemokine secreted by Toll-like receptoractivated human macrophages, and is crucially involved in sustaining their inflammatory response [4, 7]. However, the targets and functional effects of Wnt5A on human VEC remained unclear. 
Recently, we identified a novel Wnt5A signalling mechanism affecting monolayer integrity and barrier function [8] in our established in vitro system of primary immunocompetent VEC, human coronary artery endothelial cells (HCAEC) [9]. Wnt5A targets the Rho-associated protein serine/threonine kinase (ROCK) to phosphorylate LIMK2 and actin depolymerisation factor CFL1, thereby increasing actin stress fiber formation. This disrupts $\beta$-catenin and VE-cadherin at adherens junctions (AJ) leading to inter-endothelial gap formation and enhanced endothelial monolayer permeability. To mediate its effects on endothelial barrier function, Wnt5A has been found to act through Ryk receptor as silencing Ryk expression prevented Wnt5A-induced hyperpermeability [8]. Antagonising Wnt5A activity with Ryk specific Wnt inhibitory factor (WIF) -1 [10] prevents Wnt5A-induced actin stress fiber formation $[8,11]$ while Fzd or Ror specific secreted Frizzled-related peptide (sFRP) [10] is ineffective $[8,11]$.

However, the ability of the natural Wnt antagonists sFRP1 and WIF1 to antagonize LIMK/CFL phosphorylation and AJ disruption, the cellular events occurring upstream and downstream of enhanced actin stress fiber formation upon Wnt5A treatment in HCAEC need to be still verified. Here, we investigated the effects of sFRP1 and WIF1 on the phosphorylation of LIMK and CFL in human coronary artery endothelial cells (HCAEC) in the presence of Wnt5A.

\section{Methods}

\section{Cell culture}

HCAEC (Cat. No. CC-2585, Clonetics, Lonza, Switzerland) were propagated in EBM-2 medium (Clonetics, Lonza) supplemented with EGM-2MV Single Quots with 5\% FBS (Clonetics, Lonza) [9]. HCAEC used in this study were tested positively and functionally for CD31/105, von
Willebrand Factor VIII, and acetylated low density lipoprotein uptake as certified by the manufacturer (Lonza). Additionally, we confirmed stable CD31 expression by HCAEC under experimental conditions (Additional file 1: Figure S1). Cells were treated with recombinant human/ mouse Wnt5A (250 ng/mL, R\&D systems), recombinant human WIF1 (15 $\mu \mathrm{g} / \mathrm{mL}, \mathrm{R} \& \mathrm{D}$ systems) or recombinant human sFRP1 (10 $\mu \mathrm{g} / \mathrm{mL}, \mathrm{R} \& \mathrm{D}$ systems) [8]. Experiments were performed using cells from passages 3-6.

\section{Immunoblotting}

Immunoblotting of pLIMK, LIMK, pCFL and CFL and quantification of band densities were performed as described [11]. The following primary antibodies were used with dilutions indicated: rabbit anti-phospho LIMK2, polyclonal (1:500, Abcam), rabbit anti-LIMK2, polyclonal (1:1000, ThermoFischer Scientific), rabbit anti-phospho CFL, polyclonal (1:1000, Abcam), rabbit anti- CFL, polyclonal (1:1000, Abcam). Anti-rabbit IgG- HRP-linked whole antibody (GE Healthcare UK Limited) was used as the secondary antibody, diluted 1:5000.

\section{Immunofluorescence staining}

Immunofluorescence staining for $\beta$-catenin and VEcadherin were performed as described [8]. The following primary antibodies were used with dilutions indicated: rabbit-anti- $\beta$-catenin (1:150, Cell Signalling Technology), rabbit-anti-VE-cadherin (1:100, Cell Signalling Technology), rabbit-anti-CD31 (1:40, Epitomics). Alexa 568 labelled goat anti-rabbit antibody was used as the secondary antibody, diluted 1:2000. The cellular fluorescence intensities of $\beta$-catenin and VE-cadherin at inter-cellular boarders were quantified from five different fields (covering approx. 90\% of total cells/field) using ImageJ based Fiji
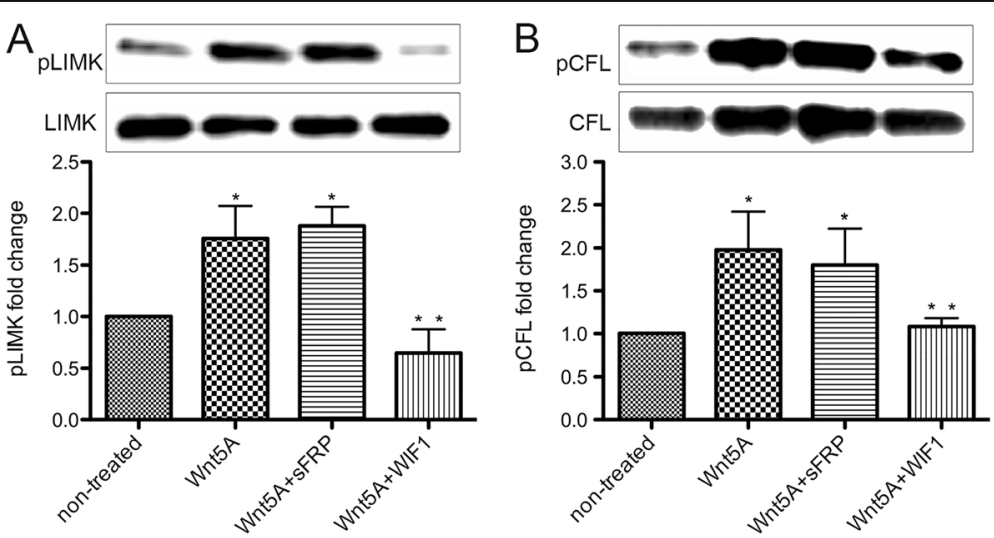

Fig. 1 Effects of the Wnt antagonists WIF1 and sFRP1 on Wnt5A-mediated phosphorylation of LIMK and CFL in VEC. Immunoblot of (a) pLIMK, LIMK and (b) PCFL and CFL in HCAEC treated with Wnt5A alone and in combination with either WIF1 or sFRP1 (sFRP) for 1 and 4 h, respectively. Bands from left to right, non-treated cells; Wnt5A-treated cells; Wnt5A + sFRP1 treated cells; Wnt5A + WIF1 treated cells. The ratios of (a) pLIMK2/ LIMK2, and (b) pCFL/CFL were calculated from densitometry analyses. Data are mean \pm SEM from three independent experiments. ${ }^{*} P<0.05$ vs non-treated, ${ }^{* *} P<0.05$ vs Wnt5A 
software (Fiji is Just ImageJ) and corrected for background fluorescence.

\section{Statistical analysis}

Data were analysed using GraphPad Prism software version 5.04 (GraphPad Software, San Diego, CA). An unpaired 2tailed Student's $t$-test or for comparing data among groups, 1-way ANOVA followed by the Newman-Keuls test was used and differences were considered statistically significant at $P<0.05$.

\section{Results and discussion}

Phosphorylation of LIMK/CFL were significantly increased in Wnt5A-treated HCAEC compared with non-treated cells (Fig. 1), thereby confirming previous findings [8]. Here, we further tested the antagonizing effects of WIF1 on Wnt5A-induced phosphorylation of LIMK/CFL. As shown herein by western blot analysis, combining Wnt5A with WIF1 significantly suppressed Wnt5A-triggered phosphorylation of both LIMK and CFL while combination with sFRP was not effective (Fig. 1). When activated by phosphorylation, LIMK phosphorylates the actin depolymerisation factor CFL1. Phosphorylation inactivates CFL1 and prevents its ability to depolymerise actin filaments leading to the formation of actin stress fibers. Increased stress fiber formation disrupts the AJ assembly at inter-endothelial junctions [12]. Here, we further investigated the antagonizing effects of WIF1 on Wnt5A-induced disruption of AJ. In non-treated cells, $\beta$-catenin and VEcadherin were aligned at the cellular periphery forming intercellular boarders. Consistent with our previous finding [8], treatment with Wnt5A notably disrupted both $\beta$ catenin and VE-cadherin at intercellular boarders, forming large inter-endothelial gaps (Fig. 2, white arrow heads). Additionally, this study has shown that combining Wnt5A with WIF1 notably prevented Wnt5A-induced disassembly of both $\beta$-catenin and VE-cadherin while sFRP failed to exert any antagonistic effects (Fig. 2, Additional file 2: Figure S2A, B). This finding is supported by functional measurements of HCAEC monolayer permeability. Treatment with Wnt5A significantly decreased the resistance of HCAEC monolayers to alternating current, thereby indicating enhanced permeability of Wnt5A-treated HCAEC monolayers (Additional file 3: Figure S3), confirming previous findings [8]. Combining Wnt5A with WIF1 significantly prevented Wnt5A-induced endothelial hyperpermeability while no significant antagonistic effect was observed with sFRP1 (Additional file 3: Figure S3).

Ryk was previously identified as the Wnt5A receptor on HCAEC [8]. The current study demonstrates the potency of WIF1 to prevent Wnt5A-induced cytoskeleton remodelling and monolayer barrier dysfunction in human vascular endothelial cells. WIF1 is a 371 amino acid protein composed of a distinct WIF domain homologous to

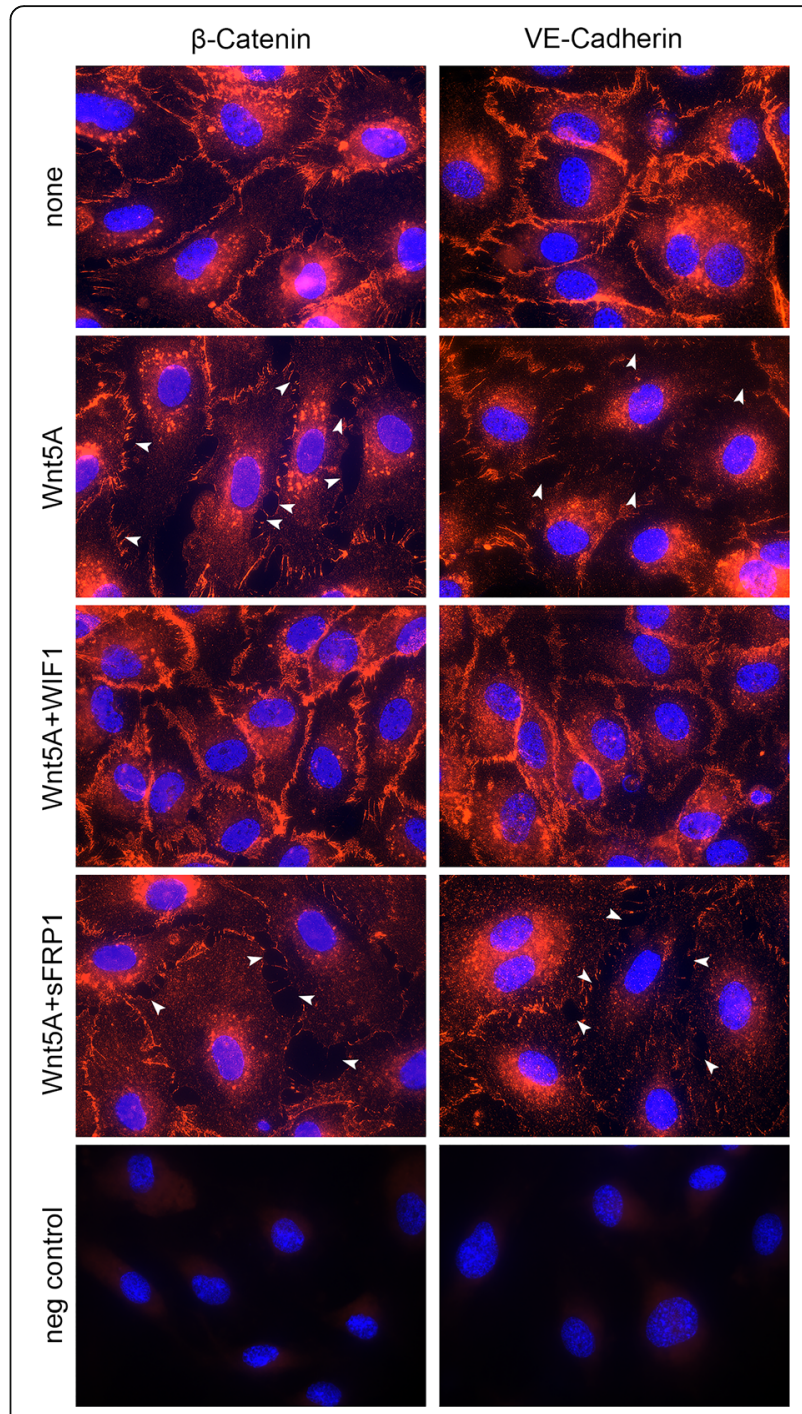

Fig. $2 \mathrm{AJ}$ assembly in VEC treated with Wnt5A in the presence or absence of WIF1 and sFRP1. Immunofluorescence staining for $\beta$ catenin and VE-cadherin (red) in HCAEC treated with Wnt5A either alone or in the presence of WIF1 and sFRP1 for $8 \mathrm{~h}$. Blue stain (DAPI) indicates nuclei. Arrow heads point to inter-endothelial gaps. Zeiss Axioskope, original magnification $630 \times$

the Wnt binding WIF domain of the Ryk receptor, five EGF-like domains, and a hydrophilic tail [13]. Secreted Wnt antagonists including WIF1 and sFRP sequester Wnt proteins away from the receptors, thereby inhibiting signalling pathways $[10,13]$ (see Scheme, Fig. 3). It has been shown that the antagonistic effect of WIF1 is mainly mediated by its WIF domain. In an attempt to map the Wnt binding sites in WIF domain, previous studies revealed the presence of an alkyl-binding site that is capable of interacting with essential lipid groups of Wnts [14-16]. Although WIF1 is expressed in different tissues, higher levels are reported in cartilage, lung, retina and brain [13, 17-20]. Several studies link elevated expression level of Wnt5A 


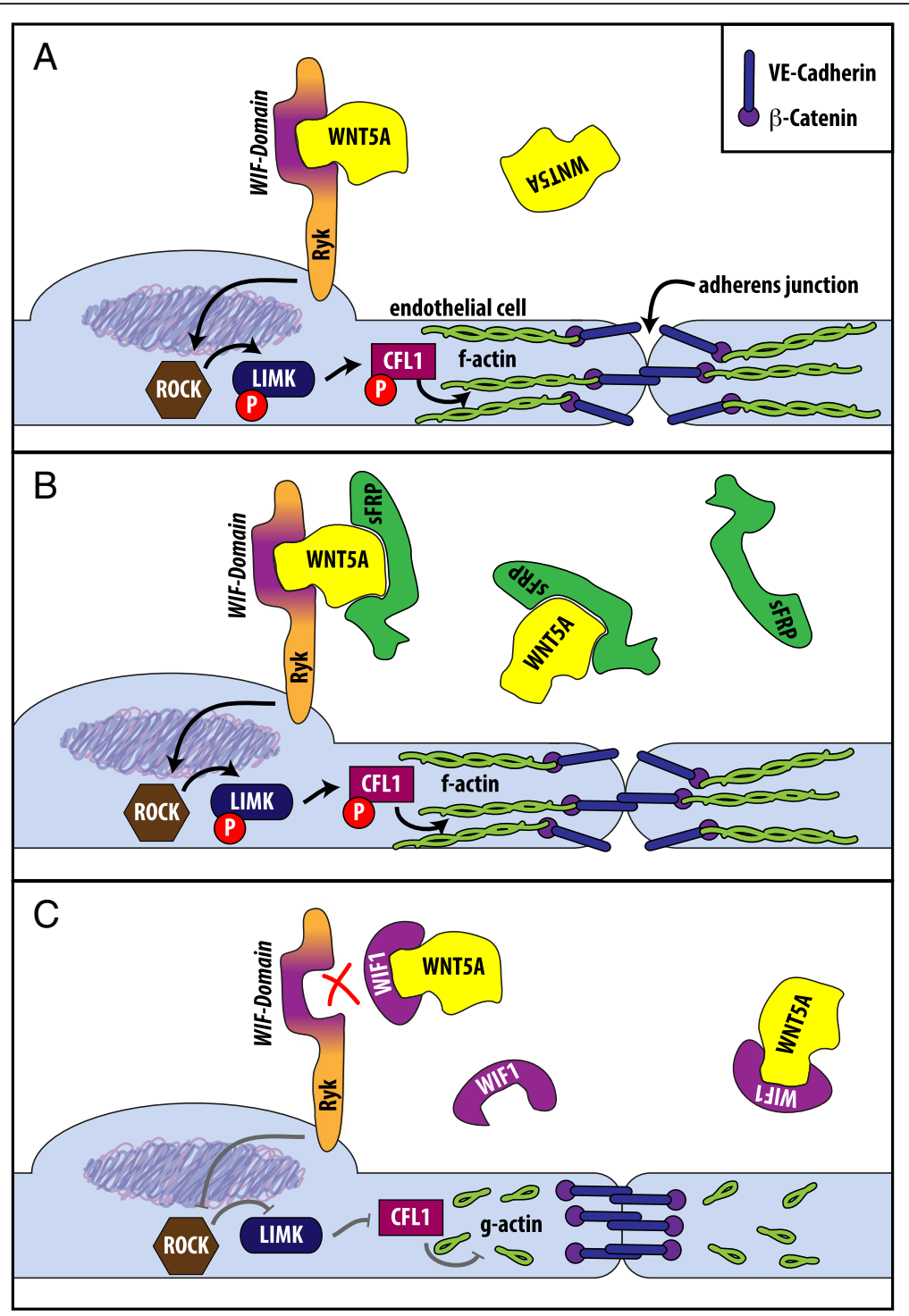

Fig. 3 Interaction of antagonists with Wnt5A/Ryk signalling in vascular endothelial cells. a Wnt5A binds to the Ryk receptor by interaction with its WIF domain, inducing downstream activation of the ROCK/LIMK/CFL pathway. Phosphorylated CFL is inactivated and allows formation of actin stress fibers (f-actin), that can interact with adherens junction protein $\beta$-catenin and tears VE-cadherin junctions apart. As a result, endothelial monolayer permeability is increased. $\mathbf{b}$ In the presence of the Wnt antagonist secreted Frizzled-related peptide (SFRP) that covers the Wnt5A binding site for the cysteine-rich domain of Frizzled receptors, interaction of Wnt5A with Ryk is still unaffected. $\mathbf{c}$ In the presence of the Wnt antagonist WIF1 (WIF) that covers the Wnt5A binding site interacting with Ryk receptor's WIF domain, Wnt5A/Ryk interaction is blocked, and downstream signalling is not transduced. CFL1 remains active and restricts f-actin formation. Actin remains mainly in the globular form (g-actin), that does not interact with adherens junction proteins

to inflammatory response in sepsis and atherosclerosis [4-6, 21]. Inflammatory activated leucocytes are the major source of Wnt5A [4, 7] and in this context, the observation that elevated Wnt5A levels correlate positively with the leucocyte count in sepsis is of particular interest [5]. The presence of lower levels of sFRP observed during worsening of disease in sepsis patients [5] is of further interest as various sFRP are shown to attenuate Fzd receptor-mediated inflammatory Wnt5A signalling in leucocytes $[4,7]$. A previous study showed the upregulation of WIF1 mRNA in pro-inflammatory activated human monocytes [4]. Presently, it is not clear if monocytes release WIF1 to counter regulate the adverse effects of Wnt5A on VEC. In light of recent in vitro findings supporting an inhibitory effect of WIF1 on Wnt5A signalling in human VEC, the altered expression and antagonistic effects of WIF1 in diseases showing dysregulated Wnt5A signalling is worthy of future investigations.

In conclusion, this is the first report identifying antagonistic effects of WIF1 on Wnt5A mediated actin cytoskeleton 
signalling pathway in primary human vascular endothelial cells. Our data suggest that the Wnt5A pathway leading to barrier dysfunction of vascular endothelial cells is a target for the natural Wnt5A antagonist WIF1. This finding could offer novel therapeutic options for diseases associated with severe vascular leakage such as sepsis and septic shock.

\section{Additional files}

Additional file 1: Figure S1. Expression of CD31 in HCAEC. Immunofluorescence staining for CD31 protein (red) in HCAEC either untreated (none) or treated with Wnt5A or TNF-alpha $(20 \mathrm{U} / \mathrm{mL})$ for $8 \mathrm{~h}$. Nuclei are stained blue (DAPI). Zeiss Axioskope, original magnification $630 \times$. (PDF $8535 \mathrm{~kb}$ )

Additional file 2: Figure S2. $\beta$-catenin and VE-cadherin expression at inter-cellular boarders. Mean fluorescence intensities of (a) $\beta$-catenin and (b) VE-cadherin at inter-cellular boarders quantified using ImageJ based Fiji software. Data are mean \pm SEM from three independent experiments. ${ }^{*} P<0.05$ vs non-treated, ${ }^{*} P<0.05$ vs Wnt5A. (ZIP $1032 \mathrm{~kb}$ )

Additional file 3: Figure S3. Barrier function of Wnt5A-treated VEC in the presence or absence of WIF1 and sFRP1. a ECIS assisted measurements (Additional file 4: supplementary methods) showing resistance of HCAEC monolayers grown in $8 \mathrm{~W} 10 \mathrm{E}+$ arrays treated with vehicle (black), Wnt5A (green), Wnt5A + WIF1 (yellow) and Wnt5A + sFRP1 (purple). Data shown are the resistance measurements conducted at $4000 \mathrm{~Hz}$ and are mean \pm SEM of 2 wells from 1 out of three representative experiments. $\mathbf{b}$ Barrier function measurements indicating the significance of WIF1's antagonistic effect on Wnt5A and are mean \pm SEM of three independent experiments run with duplicate wells. ${ }^{*} P<0.05$ vs non-treated, ${ }^{* *} P<0.05$ vs Wnt5A. (PDF $2125 \mathrm{~kb}$ )

Additional file 4: Supplementary methods. (PDF 300 kb)

\section{Abbreviations}

AJ: Adherens junctions; HCAEC: Human coronary artery endothelial cells; sFRP: Secreted frizzled-related peptide; VEC: Vascular endothelial cells; WIF1: Wnt inhibitory factor -1

\section{Acknowledgements}

We thank Jeremy Deuel, MD, PhD for drawing the scheme.

\section{Funding}

This study was supported by the Swiss National Science Foundation No. 31-124861 to Gabriele Schoedon.

\section{Availability of data and materials}

The data supporting the findings of this study are included in this article and its Additional files 1, 2, 3 and 4.

\section{Authors' contributions}

GS, TS and EB designed the research. TS and GS performed the experiments. TS, GS and EB analysed the data. TS, GS and EB wrote the manuscript. All authors read and approved the final manuscript.

\section{Authors' information}

TS: PhD, Research Scientist, Inflammation Research Unit, Division of Internal Medicine, University Hospital Zürich, Zürich, Switzerland. EB: MD, Director, Department of Medicine, Uster Hospital, Uster, Switzerland. GS: PhD, Professor of Clinical Biochemistry, Inflammation Research Unit, Division of Internal Medicine, University Hospital Zürich, Zürich, Switzerland.

\section{Competing interests}

The authors declare that they have no competing interests.

\section{Consent for publication}

Not applicable.

Ethics approval and consent to participate Not applicable.

\section{Publisher's Note}

Springer Nature remains neutral with regard to jurisdictional claims in published maps and institutional affiliations.

\section{Author details}

${ }^{1}$ Inflammation Research Unit, Division of Internal Medicine, University Hospital Zürich, Rämistrasse 100, CH-8091 Zürich, Switzerland. ²Department of Medicine, Uster Hospital, Brunnenstrasse 42, CH-8610 Uster, Switzerland.

Received: 19 January 2017 Accepted: 12 May 2017

Published online: 19 May 2017

\section{References}

1. Pober JS, Sessa WC. Evolving functions of endothelial cells in inflammation. Nat Rev Immunol. 2007;7:803-15.

2. Galley HF, Webster NR. Physiology of the endothelium. Br J Anaesth. 2004; 93:105-13.

3. Angers S, Moon RT. Proximal events in Wht signal transduction. Nat Rev Mol Cell Biol. 2009:10:468-77.

4. Pereira C, Schaer DJ, Bachli EB, Kurrer MO, Schoedon G. Wnt5A/CaMKII signaling contributes to the inflammatory response of macrophages and is a target for the antiinflammatory action of activated protein $\mathrm{C}$ and interleukin-10. Arterioscler Thromb Vasc Biol. 2008;28:504-10.

5. Schulte DM, Kragelund D, Muller N, Hagen I, Elke G, Titz A, Schadler D, Schumacher J, Weiler N, Bewig B, et al. The wingless-related integration site$5 \mathrm{a} /$ secreted frizzled-related protein- 5 system is dysregulated in human sepsis. Clin Exp Immunol. 2015;180:90-7.

6. Christman 2nd MA, Goetz DJ, Dickerson E, McCall KD, Lewis CJ, Benencia F, Silver MJ, Kohn LD, Malgor R. Wnt5a is expressed in murine and human atherosclerotic lesions. Am J Physiol Heart Circ Physiol. 2008;294:H2864-70.

7. Blumenthal A, Ehlers S, Lauber J, Buer J, Lange C, Goldmann T, Heine H, Brandt E, Reiling N. The Wingless homolog WNT5A and its receptor Frizzled5 regulate inflammatory responses of human mononuclear cells induced by microbial stimulation. Blood. 2006;108:965-73.

8. Skaria T, Bachli E, Schoedon G. Wnt5A/Ryk signaling critically affects barrier function in human vascular endothelial cells. Cell Adh Migr. 2016;1-15.

9. Franscini N, Bachli EB, Blau N, Leikauf MS, Schaffner A, Schoedon G. Gene expression profiling of inflamed human endothelial cells and influence of activated protein C. Circulation. 2004;110:2903-9.

10. Green J, Nusse R, van Amerongen R. The role of Ryk and Ror receptor tyrosine kinases in Wnt signal transduction. Cold Spring Harb Perspect Biol. 2014;6:1-12.

11. Skaria T, Burgener J, Bachli E, Schoedon G. IL-4 Causes Hyperpermeability of Vascular Endothelial Cells through Wnt5A Signaling. PLoS ONE. 2016;11:e0156002.

12. Vandenbroucke $E$, Mehta D, Minshall R, Malik AB. Regulation of endothelial junctional permeability. Ann N Y Acad Sci. 2008;1123:134-45.

13. Cruciat CM, Niehrs C. Secreted and transmembrane wnt inhibitors and activators. Cold Spring Harb Perspect Biol. 2013;5:a015081.

14. Liepinsh E, Banyai L, Patthy L, Otting G. NMR structure of the WIF domain of the human Wnt-inhibitory factor-1. J Mol Biol. 2006;357:942-50.

15. Banyai $L$, Kerekes K, Patthy L. Characterization of a Wnt-binding site of the WIF-domain of Wnt inhibitory factor-1. FEBS Lett. 2012;586:3122-6.

16. Kerekes K, Banyai L, Patthy L. Wnts grasp the WIF domain of Wnt Inhibitory Factor 1 at two distinct binding sites. FEBS Lett. 2015;589:3044-51.

17. Hsieh JC, Kodjabachian L, Rebbert ML, Rattner A, Smallwood PM, Samos CH, Nusse R, Dawid IB, Nathans J. A new secreted protein that binds to Wnt proteins and inhibits their activities. Nature. 1999:398:431-6.

18. Hunter DD, Zhang M, Ferguson JW, Koch M, Brunken WJ. The extracellular matrix component WIF-1 is expressed during, and can modulate, retinal development. Mol Cell Neurosci. 2004;27:477-88.

19. Hu YA, Gu X, Liu J, Yang Y, Yan Y, Zhao C. Expression pattern of Wnt inhibitor factor 1(Wif1) during the development in mouse CNS. Gene Expr Patterns. 2008;8:515-22.

20. Surmann-Schmitt C, Widmann N, Dietz U, Saeger B, Eitzinger N, Nakamura Y, Rattel M, Latham R, Hartmann C, von der Mark H, et al. Wif-1 is expressed at cartilage-mesenchyme interfaces and impedes Wnt3a-mediated inhibition of chondrogenesis. J Cell Sci. 2009;122:3627-37.

21. Malgor R, Bhatt PM, Connolly BA, Jacoby DL, Feldmann KJ, Silver MJ, Nakazawa M, McCall KD, Goetz DJ. Wnt5a, TLR2 and TLR4 are elevated in advanced human atherosclerotic lesions. Inflamm Res. 2014:63:277-85. 